\title{
Novel Fuzzy-Based Self-Adaptive Single Neuron PID Load Frequency Controller for Power System
}

Research Article

\author{
M. Abdullah Eissa \\ Mechanical Engineering Department, Mechatronics Division, Faculty of Engineering, \\ Helwan University, Cairo, Egypt
}

Received December 5, 2018; Accepted December 27, 2018

\begin{abstract}
This paper proposes a newly adaptive single-neuron proportional integral derivative (SNPID) controller that uses fuzzy logic as an adaptive system. The main problem of the classical controller is lacking the required robustness against disturbers, measurement noise in industrial applications. The new formula of the proposed controller helps in fixing this problem based on the fuzzy logic technique. In addition, the genetic algorithm (GA) is used to optimize parameters of the SNPID controller. Because of the high demands on the availability and efficiency of electrical power production, the design of robust load-frequency controller is becoming increasingly important due to its potential in increasing the reliability, maintainability and safety of power systems. So, the proposed controller has been applied for load-frequency control (LFC) of a single-area power system. The effectiveness of the proposed SNPID controller has been compared with the conventional controllers. The simulation results show that the proposed controller approach provides better damping of oscillations with a smaller settling time. This confirms its superiority against its counterparts. In addition, the results show the robustness of the proposed controller against the parametric variation of the system.
\end{abstract}

Keywords: Power system • Load frequency •Intelligent Control • PID • Single Neuron

\section{Introduction}

Energy is a pivotal issue that faces the world these days. Power systems have experienced a strong global growth over the last several years. In addition, power systems are considered as one of the most important application areas of control system techniques. The design of load frequency controller is essential for power system's control and operation. A robust design of controller's scheme for these systems increases the overall system's lifetime, improves the system's efficiency and reduces wasted energy. Well tuning of controllers leads the plants to still operate in a controllable region and reduces the number of shutdowns (Gupta et al., 2017; Isermann, 2006).

The main objective of control systems of industrial applications is to determine the optimum operation of a process plant by maintaining the controlled variables at the set points. Today, the three-term controller (proportional, integral and derivative) in the most feedback process controllers provides three coordinated responses to minimize the error between the measurement and the set point. The proportional term $\left(k_{p}\right)$ adjusts the input signal by an amount proportional to the amount of error. The derivative term $\left(k_{d}\right)$ adds to improve the overshoot of the process response. To eliminate the steady-state error, the integral term $\left(K_{i}\right)$ should be inserted. The desired overall response of the target process plant is obtained by adjusting the gains of $k_{p}, k_{i}$ and $k_{d}$ (Martin and Hale, 2010; Blevins and Nixon, 2011).

Proportional integral derivative (PID) controllers are widely used in many aspects of industrial applications due to their simple structure, robust performance and easy tuning. Tuning the controller parameters is very important for the effective operation of a process. Several tuning methods have been proposed for PID controllers. The classical 
tuning methods can be classified into two main categories: open loop and closed loop. The closed-loop tuning techniques are used in an automatic mode when the plan is operating in a closed loop. In contrast, the open-loop techniques are used in a manual mode when the plant operates in an open-loop state. Ziegler-Nichols method, Tyreus-Luyben method, damped oscillation method, Cohen and Coon method, Fertik method and CianconeMarline method are different types of both tuning methods (Gupta et al., 2017; Shahrokhi and Zomorrodi, 2013).

Recently, the use of intelligent control (IC) systems has rapidly increased in many different industrial applications. Many artificial intelligence techniques such as fuzzy logic, neural networks and GA are widely applied to solve the control problems. However, there will be no observed improvement in the performance of the control system, if the IC system used is a conventional controller. So, the IC system could be used as a compliment for the conventional controllers to generating a new controller that is stable for many industrial applications. Thus, the intelligent controller that is generated from these combinations will have new features. These features are as follows: 1) flexibility - where they can adapt themselves against the environment changing and 2) learning capability where they can improve their performance based on their experience (Ponce-Cruz et al., 2009). Proceeding from this, our work combines the neural network and fuzzy logic and the traditional PID to generate a new controller called fuzzy-based self-adaptive SNPID.

Duman et al. (2012) have proposed optimal proportional integral (PI) and PID using a new gravitational search algorithm for load-frequency control (LFC) of a single-area power system. Guha et al. (2016) have demonstrated the use of grey wolf optimization method to optimize the PID controller parameters to solve the LFC problem of the two-area power system. Murat et al. have studied the application of artificial neural network to load-frequency controller of a single-area power system. Xiao-dan et al. (2017) have achieved rolling tension control by a SNPID controller. Hu et al. (2012) have proposed a new SNPID controller based on the Levenberg-Marquardt algorithm to overcome the drawbacks of the traditional SNPID controllers.

In this work, we demonstrate the capability of a SNPID controller for LFC of a single-area power system. First, we present a new design for the SNPID controller structure based on fuzzy logic. Second, we demonstrate the ability of the proposed controller to solve the problem of the LFC of a power system towards different conditions.

The remainder of the paper is organized as follows: Section 2 provides the modelling of a single-area power system. The design of the proposed SNPID for LFC is demonstrated in Section 3. Results and analysis are presented in Section 4. In Section 5, the presented controller and comparison of different controller techniques are discussed. Finally, conclusion and future work are provided in Section 6.

\section{Power system model}

Several nonlinear models for large power systems have to be established but all are complicated models. So, the linearized model has been used in our work (Bensenouci et al., 2010). The power system is represented by an equivalent turbine, governor and generator system. The transfer function of a single-area power system model controlled using the proposed SNPID controller is shown in Figure 1.

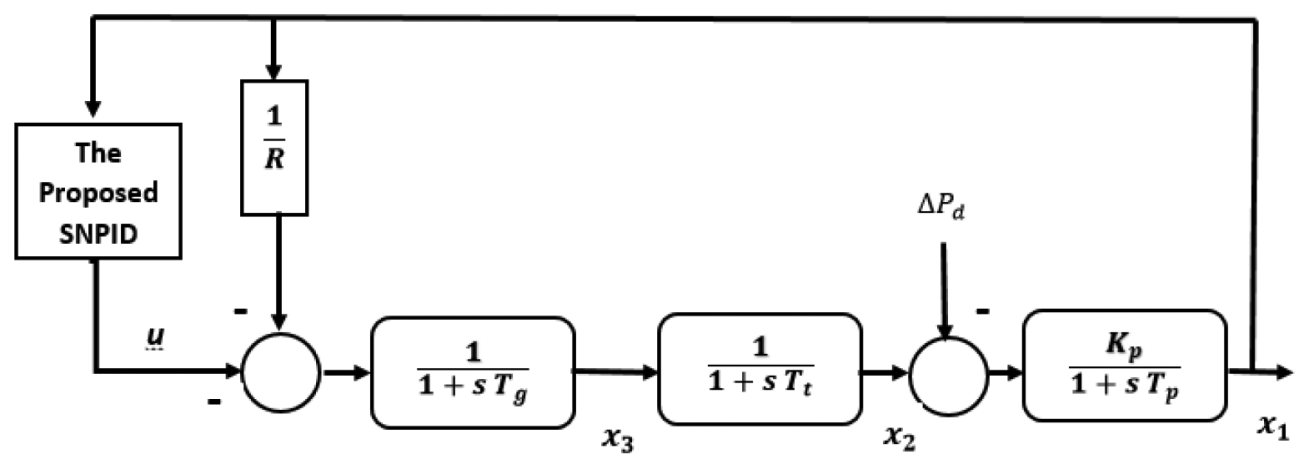

Fig 1. Block diagram of a single-area power system 
where $x$ denotes state vector $(3 \times 1), y$ output $(3 \times 1), u$ control vector $(1 \times 1), d$ disturbance vector $\left(\Delta P_{d}\right), T_{p}$ plant model time constant, $T_{t}$ turbine time constant, $T_{g}$ governor time constant, $k_{p}$ plant gain, $R$ speed regulation due to governor action, $x_{1}$ change in system frequency, $x_{2}$ incremental changes in generator output and $x_{3}$ governor valve position.

Table 1 presents the values of system parameters that are used in this work.

Table 1. System parameters

\begin{tabular}{lc}
\hline Parameters & Value \\
\hline \hline$K_{p}$ & $120 \mathrm{pu}$ \\
$T_{p}$ & $20 \mathrm{~s}$ \\
$T_{t}$ & $0.3 \mathrm{~s}$ \\
$T_{g}$ & $0.08 \mathrm{~s}$ \\
$R$ & $2.4 \mathrm{~Hz} / \mathrm{pu} \cdot \mathrm{MW}$ \\
\hline
\end{tabular}

\section{The proposed load-frequency controller design}

This work presents a new SNPID load-frequency controller for a single-area power system. SNPID is a simple neural network that consists of one neuron and has the ability to perform the function of PID controller. The structure of the SNPID controller has been illustrated in Fig 2. The SNPID controller can be expressed as:

$$
\begin{aligned}
& u(k)=u(k-1)+k_{n} \sum \bar{w}_{l}(k) x_{i}(k) \\
& w_{l}(k)=w_{i}(k) / \sum\left|w_{i}(k)\right|
\end{aligned}
$$

where $x_{1}, x_{2}$ and $x_{3}$ are the inputs of SNPID. $\mathrm{U}$ is the controller output. $w_{1}, w_{2}$ and $w_{3}$ are the neuron weights.

According to Equation 1, there are seven parameters that must be tuned to design the SNPID controller. These parameters are as follows:

- $\quad k$ is called neuron proportion coefficient.

- $\eta_{p}, \eta_{I}$ and $\eta_{D}$ are the three learning speed parameters.

- $w_{1}, w_{2}$ and $w_{3}$ are the three corresponding weights of neuron's input vector.

In this paper, the genetic algorithm (GA) has been used to determine the optimal values for these parameters. The proposed controller used fuzzy logic to update the weighted coefficients, and the control signal is generated based on a new formula, which is expressed as:

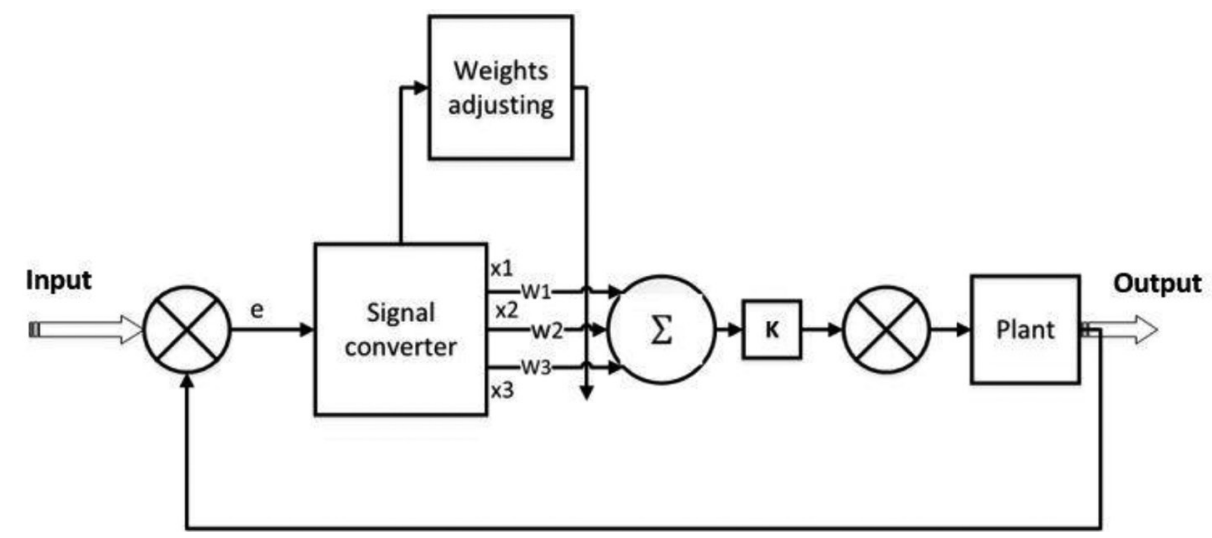

Fig 2. Block diagram of SNPID structure 


$$
\left.u=k\left[\left(k_{P f} * w_{1}(k) * e\right)+\left(k_{I f} * w_{2}(k) * \int e\right)\right)+\left(k_{D f} * w_{3}(k) * \frac{\partial e}{\partial t}\right)\right]
$$

where $k_{P f}, k_{I f}$ and $k_{D f}$ are the outputs of the fuzzy controller. The new proposed SNPID controller structure of the proposed method is shown in Fig 3.

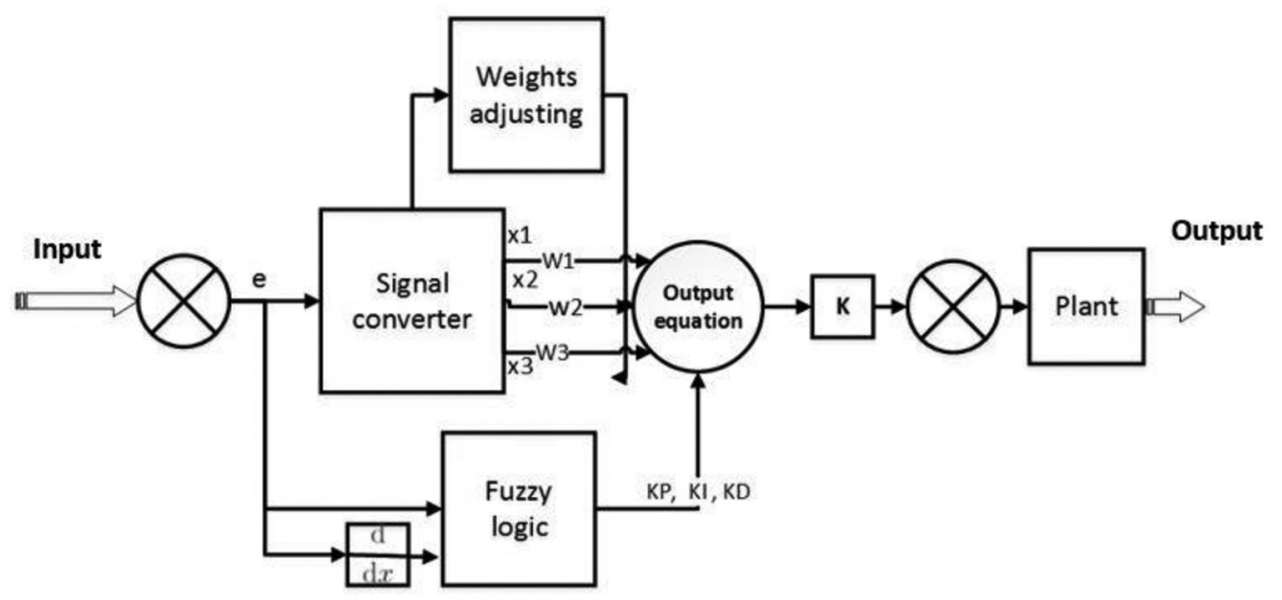

Fig 3. Block diagram of proposed fuzzy-based self-adaptive SNPID structure

Different weight-learning algorithms based on the learning theory of neural network were used to adjust the weights of SNPID controllers. Non-supervised Hebb learning rule, supervised delta learning rule, supervised Hebb learning rule and improved Hebb learning rule are the popular four weight-learning algorithms that are used. In the proposed method, the four weight-learning algorithms are used based on the equations presented in Wang et al. (2015). For the fuzzy logic system, the membership function adopts triangle function, the fuzzy inference adopts Madami rules and the defuzzification adopts a weighted average method, and the fuzzy rule tables of $k_{P f}, k_{I f}$ and $k_{D f}$ have been presented in Wang et al. (2015).

\section{Results and analysis}

This section presents the simulation results of implementing the proposed control algorithms and compared them with those of other traditional controllers. These controllers are classical, fuzzy self-tuning SNPIDs. The results are divided into two cases. The normal case applied two types of the disturbances. These disturbances are $\Delta P_{d 1}$ as shown in Fig 4 and $\Delta P_{d 2}$ as shown in Fig 5. $\Delta P_{d}$ simulates the realistic of the power demand changing in the power system. Case 2 is parametric uncertainty where the operating point is changed to test the power of the proposed method against its counterparts.

\subsection{Normal case}

Two experiments were implemented at the normal operating condition. In the first experiment, $\Delta P_{d 1}$ is applied to the system. Fig 6 shows the time response of the power plant. The controller's output signals for the second experiment are shown in Fig 7. Fig 8 shows the time response of the system when $\Delta P_{d 2}$ is applied to the system, and the controller's output signals are shown in Fig 9. According to these results, the proposed SNPID controller gives a better performance with a smaller settling time, acceptable undershoot. In addition, the proposed controller recovers to zero steady-state error after a smaller time delay.

\subsection{Parametric uncertainty case}

In this case, the parametric uncertainties of the power system have to be considered. According to load variation and power system configuration, the operation points of the system are changed randomly during a daily cycle. The system parametric uncertainties are obtained by changing parameters by $50 \%$ of their normal values according to Table 2 (Sakhavati et al., 2011; Bensenouci et al., 2010). In the first experiment, $\Delta P_{d 1}$ includes the effect of the parametric uncertainties applied to the system. The system response is shown in Fig 10, and the controller's outputs are shown 
in Fig 11. The second experiment was conducted under the influence of both $\Delta P_{d 2}$ and parametric uncertainties. Fig 12 shows the time response of the system. In addition, the controller's output signals are shown in Fig 13. According to these results, it is clear that the proposed controller has the best response compared to other controllers. In addition, the results approve the effectiveness and the ability of the proposed controller against the parametric uncertainties.

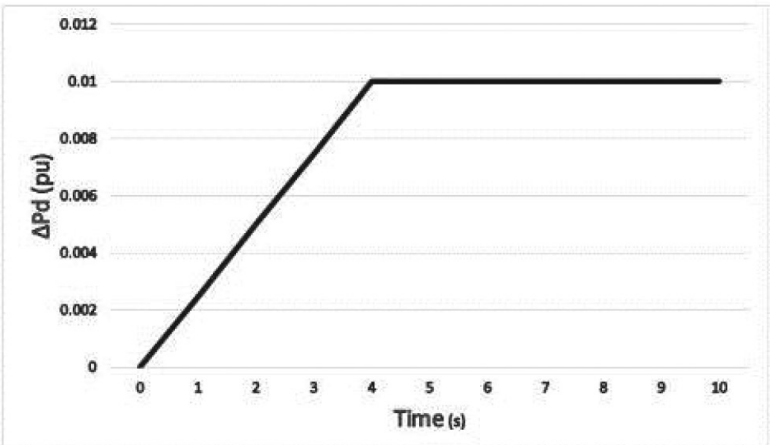

Fig 4. $\Delta P_{d 1}$

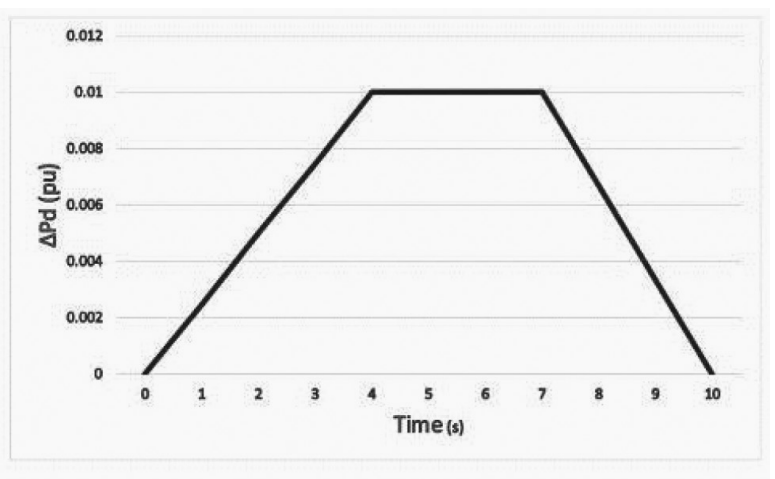

Fig 5. $\Delta P_{d 2}$

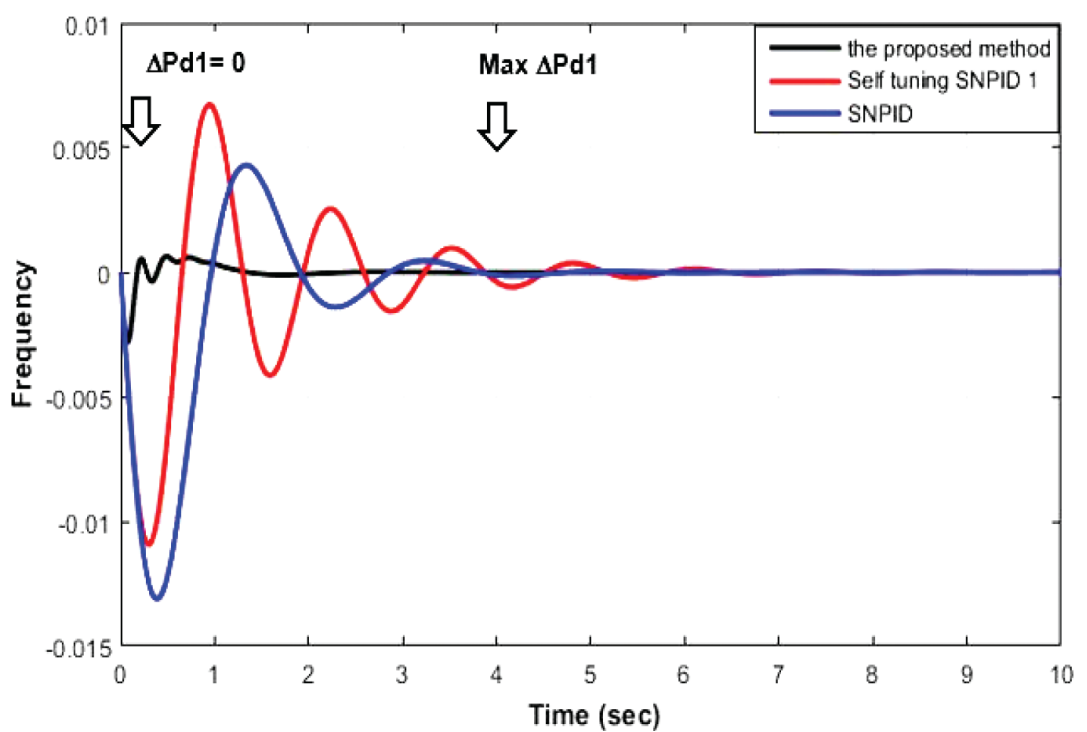

Fig 6. System dynamic responses at $\Delta P_{d 1}$ 


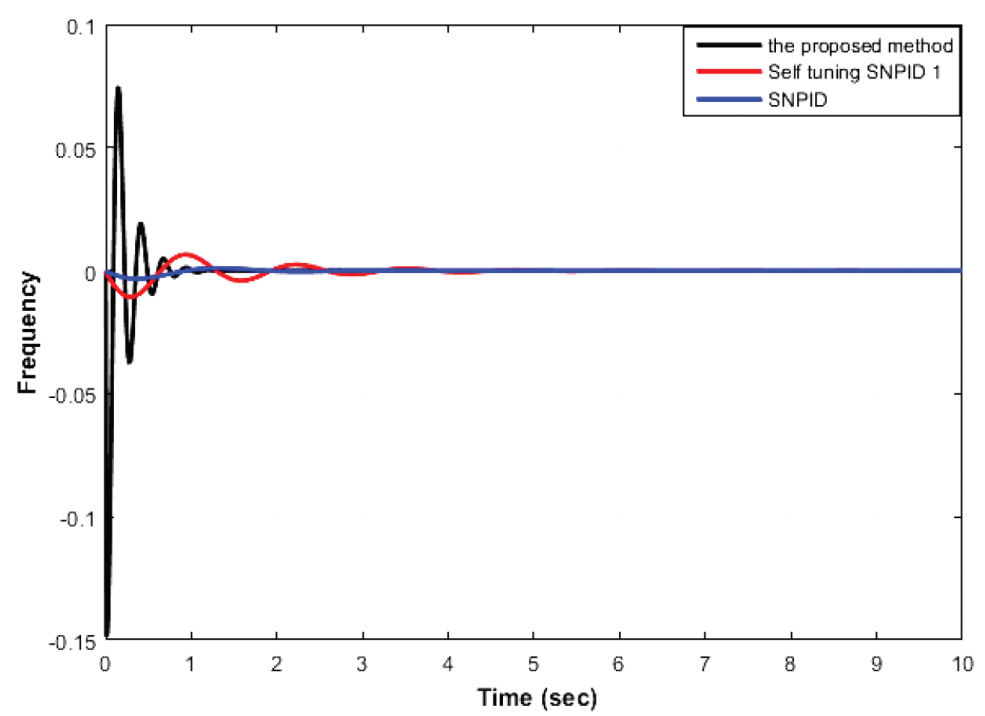

Fig 7. The controller's output signals at $\Delta P_{d 1}$

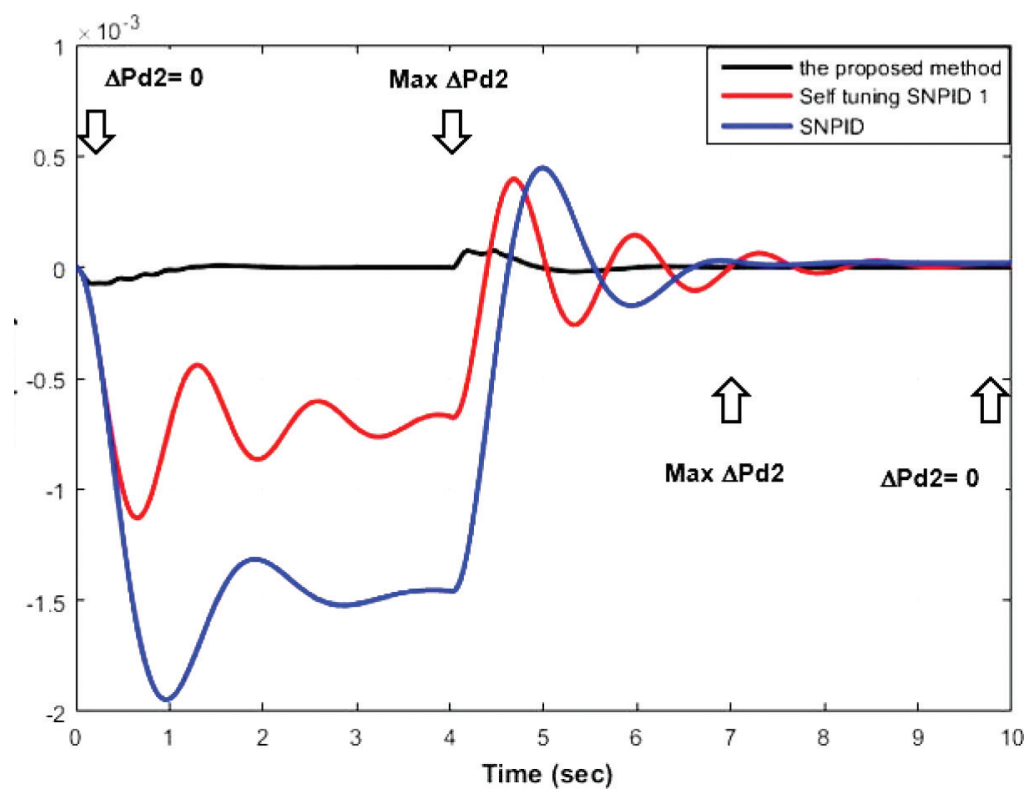

Fig 8. System dynamic responses at $\Delta P_{d 2}$ 


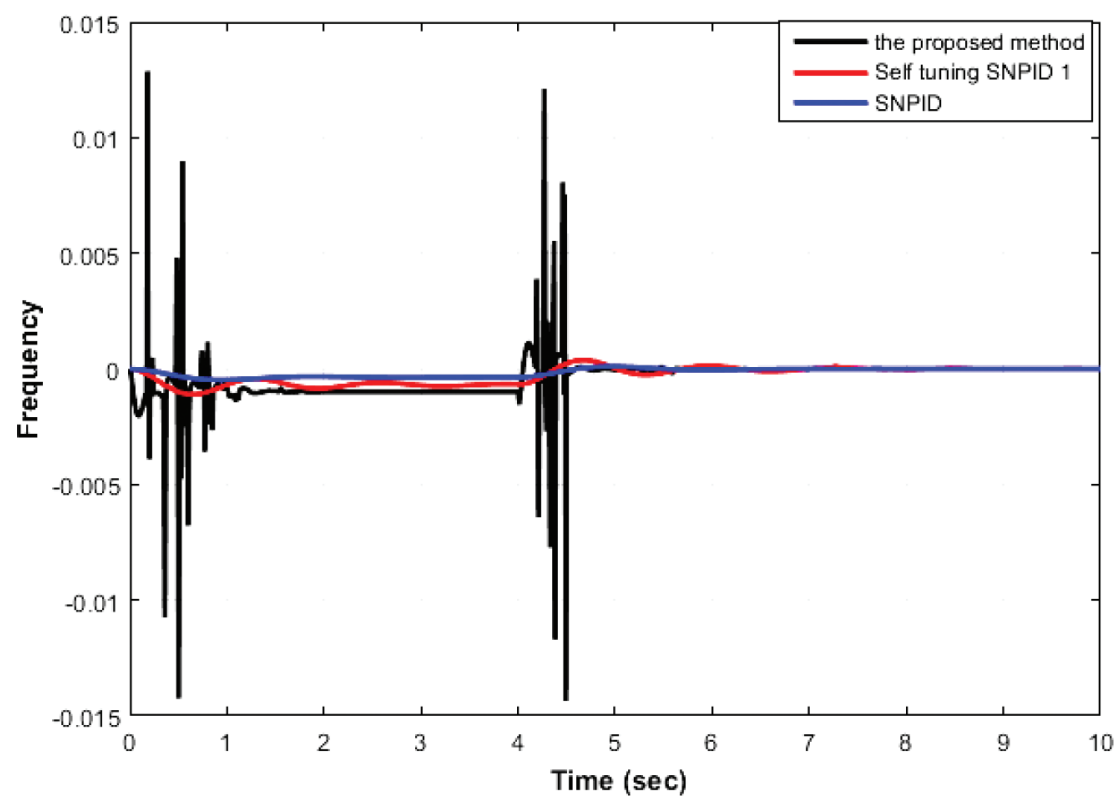

Fig 9. The controller's output signals at $\Delta P_{d 2}$

Table 2. Parametric uncertainties

\begin{tabular}{lccc}
\hline Parameters & Down & Normal & Up \\
\hline \hline$K_{p}$ & 60 & 120 & 180 \\
$T_{p}$ & 10 & 20 & 30 \\
$R$ & 1.2 & 2.4 & 3.6 \\
$T_{t}$ & 15 & 0.3 & 0.45 \\
$T_{g}$ & 0.04 & 0.08 & 0.12 \\
\hline
\end{tabular}

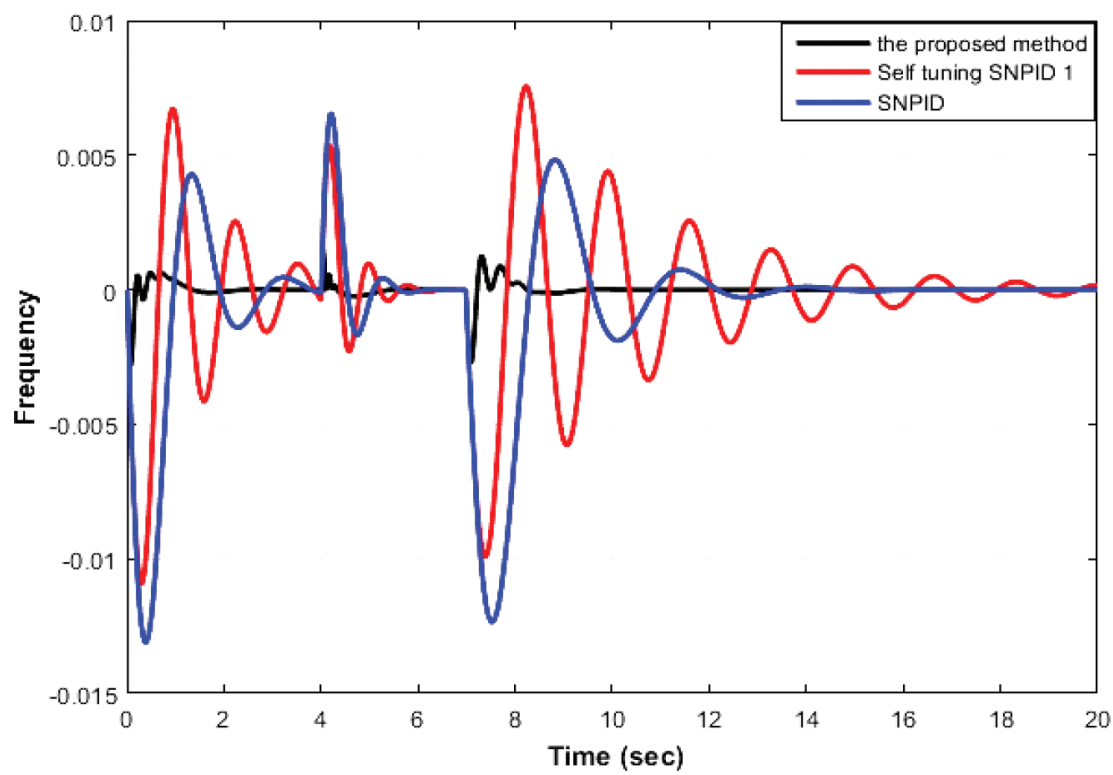

Fig 10. System dynamic responses at $\Delta P_{d 1}$ and parametric uncertainties 


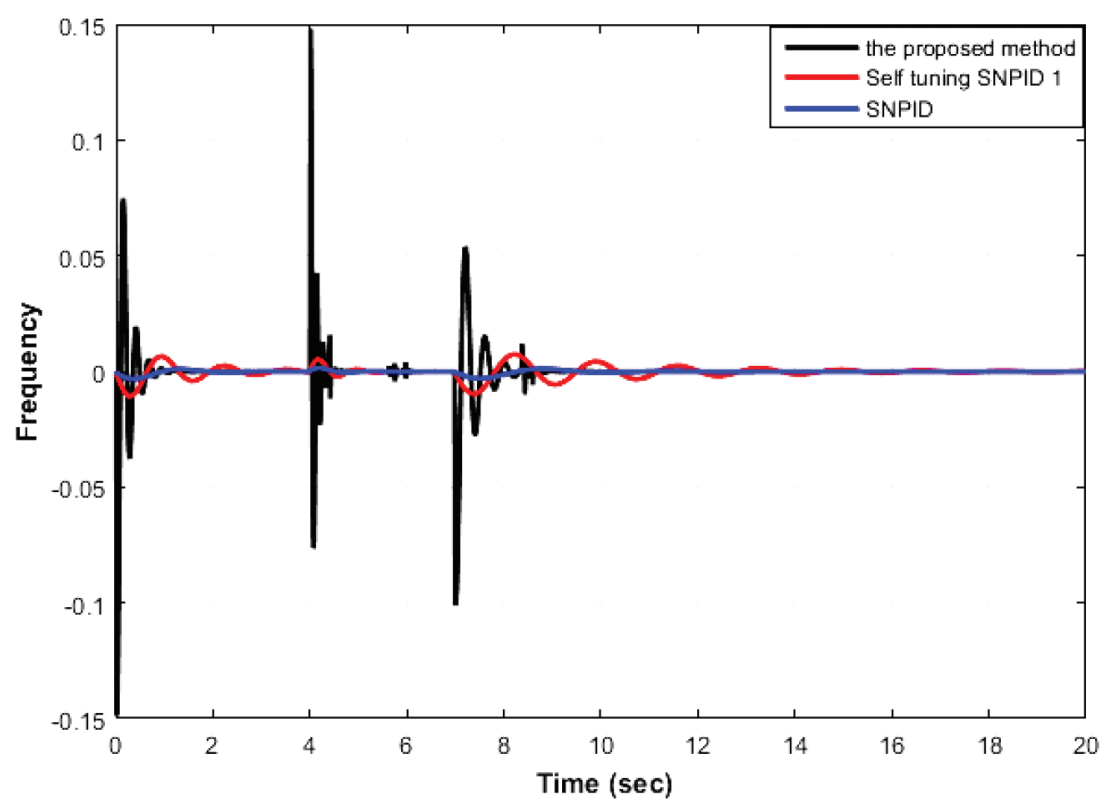

Fig 11. The controller's output signals at $\Delta P_{d 1}$ and parametric uncertainties

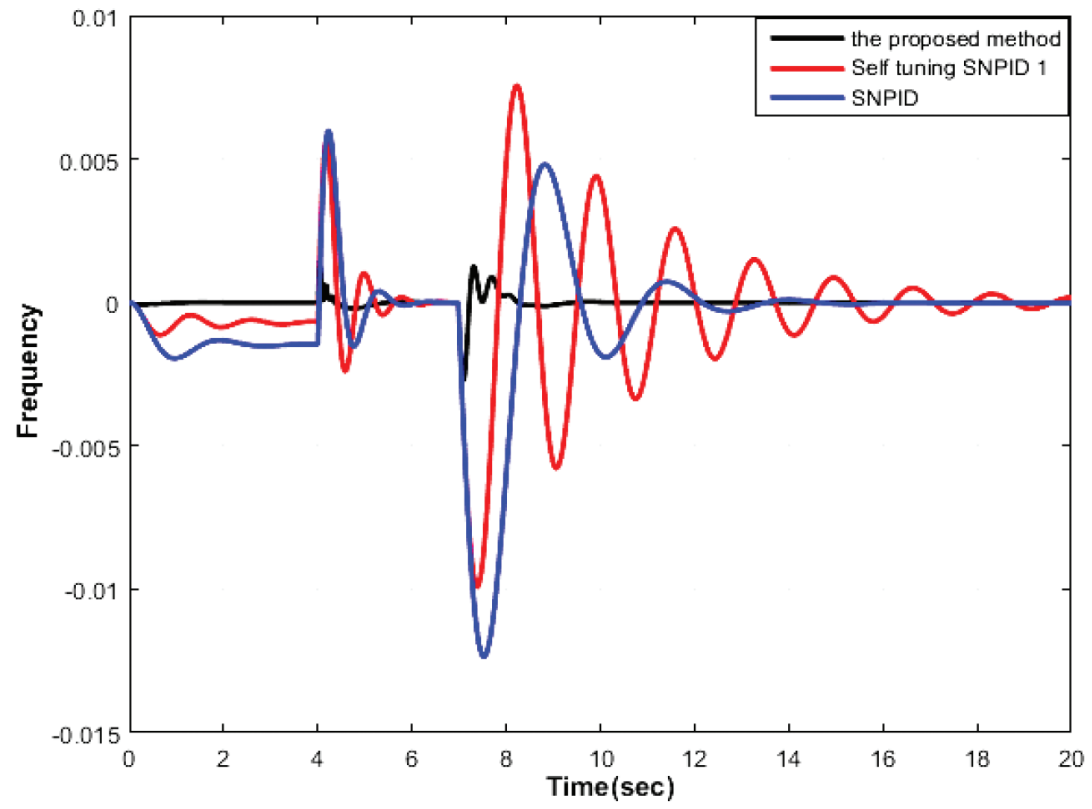

Fig 12. System dynamic responses at $\Delta P_{d 2}$ and parametric uncertainties 


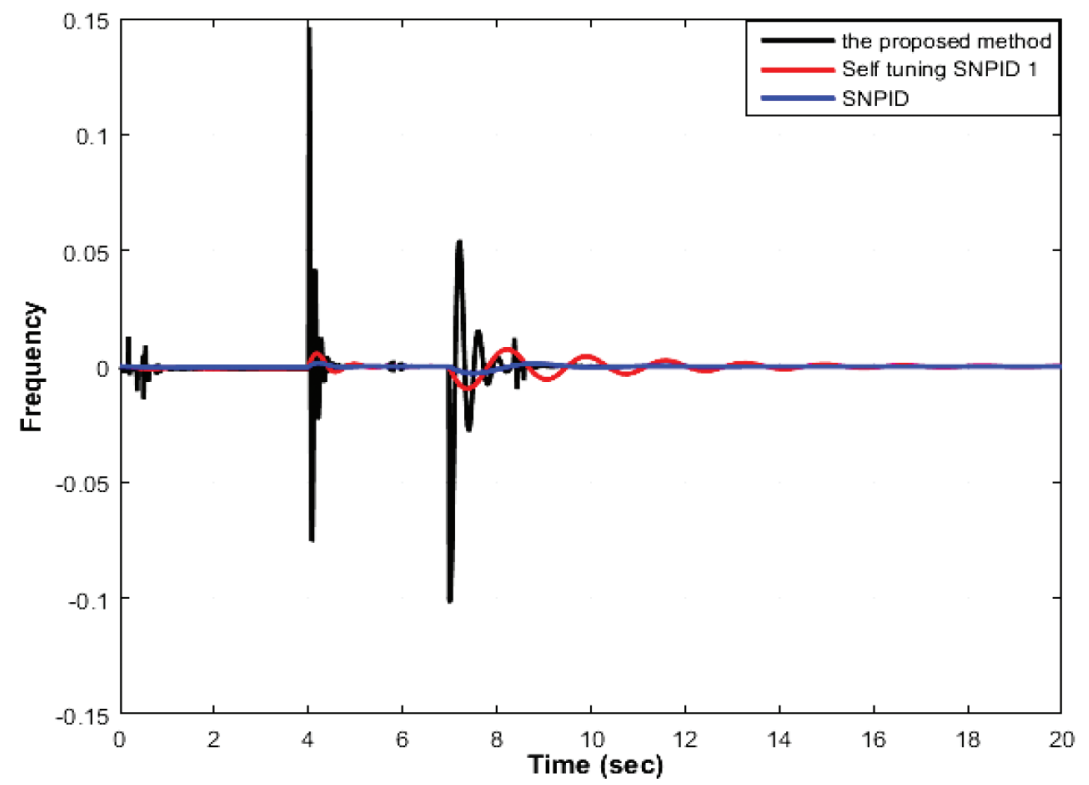

Fig 13. The controller's output signals at $\Delta P_{d 2}$ and parametric uncertainties

\section{Comparative study}

In this study, three controller algorithms have been designed with implementation on a single-area power system, namely, SNPID, self-tuning SNPID, and the proposed SNPID controller. Two types of disturbances were applied to the power system: $\Delta P_{d 1}$ and $\Delta P_{d 2}$. In addition, the system parameters were changed by $50 \%$ of their normal values to test the controller's performances under parametric uncertainties. On another side, four traditional controllers were applied to solve the same LFC problem of a single-area power system that was published in Bensenouci et al. (2010). These controllers were 1) an iterative PID (IPID), 2) an IPID based on $H_{\infty}$ (IPIDHI), 3) an IPID that was characterized by the maximum regulated output of the closed-loop system with its command input being bounded (IPIDM) and 4) an IPID based on H2 (IPIDH2). To show the advantages of the proposed controller, this section presents a comparison study between the proposed controller and six other controllers. Two of these controllers have been designed in the present work, and the other four controllers have been published in Bensenouci et al. (2010). Table 3 summarizes the comparison between these techniques. The following comparison parameters have been taken:

1. settling time,

2. undershoot and

3. steady-state error.

Table 3. Comparison of controllers

\begin{tabular}{|c|c|c|c|}
\hline \multirow[t]{2}{*}{ Controllers } & Settling time (s) & Undershoot & Stead-state error \\
\hline & \multicolumn{3}{|c|}{ Normal case at $\Delta P_{d}$} \\
\hline IPID & 3.5 & -0.077 & 0 \\
\hline IPIDHI & 2.25 & -0.053 & 0 \\
\hline IPIDH2 & 2.25 & -0.072 & 0 \\
\hline IPIDM & 2 & -0.048 & 0 \\
\hline SNPID & 4 & -0.013 & 0 \\
\hline Self-tuning SNPID & 6 & -0.011 & 0 \\
\hline Proposed controller & 1.7 & -0.002 & 0 \\
\hline
\end{tabular}


Thus, the proposed new fuzzy-based self-adaptive SNPID controller shows the best responses in all tests, and the effectiveness of this controller was carried out for diverse disturbances and parametric uncertainties. The results prove that the proposed controller satisfies the system stability and the robustness. The main advantages of the proposed controller over the other controllers are as follows: 1) fast recovery to zero steady-state error after a smaller time delay with a minimal undershoot, 2) decoupling disturbances and parametric uncertainties and 3) flexibility and self-adaptation based on the fuzzy system.

\section{Conclusion and future work}

We demonstrate a new controller called fuzzy-based self-adaptive SNPID. The proposed controllers were used for LFC of a single-area power system. First, we present the linearized model for a single-area power system, which consists of turbine, governor and generator. Second, the design of the proposed SNPID load-frequency controller has been presented where the GA was employed to determine the optimal SNPID parameters. This controller was designed using a new formula based on fuzzy logic, which was used to dynamically adjust the proportional, integral and derivative learning rates. We also validated the effectiveness of the proposed approach under normal and parametric uncertainties. The proposed method achieved the best performance compared to other traditional methods. Part of our future work is to test the proposed controller in a multi-area power system.

\section{References}

Bensenouci, A. and Ghany, A.A., (2010). Performance analysis and comparative study of LMI-based iterative PID load-frequency controllers of a singlearea power system. Power, 4, p.7.

Blevins T. and Nixon, M. (2011). Control Loop Foundation Batch and Continuous Processes. North Carolina: International Society of Automation.

Duman, S., Yorukeren, N. and Altas, I. H. (2012). Load frequency control of a single area power system using Gravitational Search Algorithm. International Symposium on Innovations in Intelligent Systems and Applications, pp. 1-5.

Guha, D., Roy, P.K. and Banerjee, S., (2016). Load frequency control of interconnected power system using grey wolf optimization. Swarm and Evolutionary Computation, 27, pp. 97-115.

Gupta, M., Walia, A., Gupta, S. and Sikander, A., (2017). Modelling and identification of single area power system for load frequency control. In: 4th IEEE International Conference on Signal Processing, Computing and Control (ISPCC), (pp. 436-439), September 2017.

Hu, T.T., Zhuang, Y.F. and Yu, J., (2012). An improved single neuron adaptive PID controller based on levenberg-marquardt algorithm. In: International Conference on Brain Inspired Cognitive Systems, (pp. 288-295). Berlin, Heidelberg: Springer.

Isermann, R. (2006). Fault-Diagnosis Systems. An Introduction from Fault Detection to Fault Tolerance. Berlin-Heidelberg: Springer.
Lüy, M., Kocaarslan, I., Çam, E., \& Taplamacioğlu, M. C. (2008). Load frequency control in a single area power system by artificial neural network (ANN). In 4 International Conference on TPE (pp. 26-29).

Martin, P.G. and Hale, G. (2010). Automation Made Easy Everything you Wanted to Know About Automation and Need to Ask. North Carolina: International Society of Automation.

Ponce-Cruz, P. and Ramírez-Figueroa, F.D. (2009). Intelligent Control Systems with LabVIEW'TM. Berlin: Springer Science \& Business Media.

Sakhavati, A., Gharehpetian, G. and Hosseini, S.H. (2011). Decentralized robust load-frequency control of power system based on quantitative feedback theory. Turkish Journal of Electrical Engineering \& Computer Sciences, 19(4), pp. 513-530.

Shahrokhi, M. and Zomorrodi, A., 2013. Comparison of PID controller tuning methods. Department of Chemical \& Petroleum Engineering Sharif University of Technology, pp.1-2.

Wang, Q., Shuang, Y. (2015). A single neuron PID control algorithm of memristor-based. Computer Information System, 14, no. 20143104, pp. 50235030 .

Xiao-dan, et al. (2017). Application of single neuron adaptive PID approach in rolling tension control. In: 2nd International Conference on Materials Science, Machinery and Energy Engineering (MSMEE), 2017. 